\title{
Molecular Targets for the Testing of COVID-19
}

\author{
Suh Kuan Yong ${ }^{1}$, Ping-Chia Su${ }^{1}$, and Yuh-Shyong Yang ${ }^{1}$ \\ ${ }^{1}$ Affiliation not available
}

April 28, 2020

The pandemic outbreaks of coronavirus disease 2019 (COVID-19) was first discovered in Wuhan, Hubei, China in December 2019. The COVID-19 was caused by the novel coronavirus, namely severe acute respiratory syndrome coronavirus 2 (SARS-CoV-2). It took 30 days to spread to all provinces of China [1]. Recently, the confirmed cases of COVID-19 have been reported from about 200 countries or regions on March 30, 2020, and killed almost 30 thousand people [2]. Efficient identification of the infection by SARS-CoV-2 has been one of the most important tasks to facilitate all the following counter measurements in dealing with infectious disease. In Taiwan, a COVID-19 Open Science Platform adhering to the spirit of open science: sharing sources, data, and methods to promote progress in academic research while corroborating findings from various disciplines has established in mid-February 2020, for collaborative research in support of the development of detection methods, therapeutics, and a vaccine for COVID-19. Research priorities include infection control, epidemiology, clinical characterization and management, detection methods (including viral RNA detection, viral antigen detection, and serum antibody detection), therapeutics (neutralizing antibody and small molecule drugs), vaccines, and SARS-CoV-2 pathogenesis. In addition, research on social ethics and the law are included to take full account of the impact of the COVID-19 virus.

\section{The virus}

The SARS-CoV-2 was named because it shares $88 \%$ nucleotide similarity to two bat-derived severe acute respiratory syndrome (SARS)-like coronaviruses and 79\% similarity to SARS-CoV that appeared in 2003 which affected 26 countries and killed about 800 people [3, 4]. There are 4 common types of human coronavirus that have been reported such as HKU1, NL63, 229E, and OC43 that cause the mild symptom of common flu, whereas SARS-CoV, Middle East respiratory syndrome-related coronavirus (MERS-CoV) and novel SARS-CoV-2 were reported that cause the severe or fatal symptom. The coronavirus is an enveloped, non-segmented positive-sense and single-stranded RNA virus with a crown-like appearance. Figure 1 shows the coronavirus structure that composes four types of protein, such as spike glycoprotein (S), envelope protein (E), membrane protein $(\mathrm{M})$ and nucleocapsid protein $(\mathrm{N})$. The $\mathrm{S}$ protein is playing an important role in invading into the host, by interacting with human receptor angiotensin-converting enzyme 2 (ACE2). The receptor-binding domain (RBD) of S protein contributed its immunogenic that attracted many neutralizing antibodies [5,6]. They are all potential molecular targets for the identification of SARS-CoV-2 infection. Currently, there is no specific therapeutic drug or vaccine for COVID-19, therefore it is necessary to deploy a robust diagnostic methodology which is rapid, accurate and ultra-sensitive to isolate infected patients from the healthy population.

\section{Viral RNA}

The testing of SARS-CoV-2 is grouped into molecular testing and serology testing. The molecular testing includes nucleic acid amplification test (NAAT) such as real-time RT-PCR [7]. According to WHO interim guidance on laboratory testing of COVID-19 in suspected human [7], the respiratory materials were required to collect to perform NAAT. The unique sequence RNA of the virus including nucleocapsid (N), envelope (E), spike protein (S) and RNA-dependent RNA-polymerase (RdRP) genes are targeted to analyze using 
real-time reverse-transcription polymerase chain reaction (rRT-PCR) [8]. On $23^{\text {rd }}$ January 2020, the first COVID-19 detection kit using rRT-PCR was published which targeted RdRP, N and E gene of SARS-CoV2 [8]. Up to present, there are many in-house or commercial COVID-19 molecular assays available from worldwide, for instance, 1. 'CDC 2019-nCoV real-time reverse transcriptase PCR diagnostic panel' was developed by United State Centers for Disease Control and Prevention (US CDC). This diagnostic panel is working with Applied Biosystems 7500 Fast DX Real-Time PCR Instrument with SDS 1.4 software; 2. 'New coronavirus nucleic acid assay' which targeted on ORF1ab and $\mathrm{N}$ gene was developed by Chinese National Institute for Viral Disease Control and Prevention; 3. Molecular test kits from four companies such as Seegene Inc., Kogene Biotech Co. Ltd., Sd Biosensor Inc., and Solgent Co. were approved by Ministry Food and Drug Safety (MFDS) and Korea Centers for Disease Control and Prevention (KCDCP) which are now widely being used in South Korea [9]. Primer and probe design are critical for the detection of or viral RNA that is easy to mutate. Taiwan Centers for Disease Control provides real-time RT-PCR primers targeting E, RdRp and N gene for first-line screening, confirmatory and additional confirmatory assay, respectively.

The outbreaks of the COVID-19 pandemic have led to the increasing of thousands of active cases every day all over the world, so worldwide are racing against time to find a test to quickly detect the virus and isolate the infected patient from healthy population to stop virus transmission from the further outbreak. The United States Food and Drug Administration (US FDA) is moving fast to approve a few COVID-19 molecular test kits under Emergency Use Authorization (EUA) powers. Cepheid Inc, Mesa Biotech Inc and BioFire Defense LLC were recently granted FDA approval of their rapid diagnostic test that could give the test result reduced to an hour compared to traditional RT-PCR methods that required 3 to 4 hours. On March 27, 2020, Abbott announced the launch of a molecular point-of-care test, which uses an isothermal RNA amplification method to deliver positive results in five minutes and negative results in 13 minutes. It is expected to produce about 5 million tests per month (https://www.abbott.com/corpnewsroom/productand-innovation/detect-covid-19-in-as-little-as-5-minutes.html).

\section{Viral Proteins}

In addition to its RNA, the $\mathrm{S}$ and the $\mathrm{N}$ proteins are frequent targets for the detection of conorovirus. In contrast to that of the RNA, the development of a detection kit on viral proteins is still in progress. A probe, usually a specific antibody, is needed before a successful viral protein detection method can be developed. In early March 2020, Academia Sinica, Taiwan reported the first monoclonal antibody against N protein of Sars-CoV-2. With their antibody library in hand, the team screened against $\mathrm{N}$ proteins from 7 different viral species to obtain one antibody against only SARS-CoV-2 N protein, 9 against only SARS-CoV N protein, and 36 against both SARS-CoV-2 and SARS-CoV N proteins [10]. The results indicated that rapid immunebased test kit of SARS-CoV-2 coronavirus can be developed in the future. It also shows the complexity and time consuming to obtaining and screening an antibody for an unbiased test targeting specific viral protein. The procedure for the preparation of specific antibody from laboratory animal has been very well established and it could be expected that numerous antibodies specific for a SARS-CoV-2 protein will be reported soon. Twenty-five strains of antibodies from 3 COVID-19 patients have also been prepared from Chang Gung Memorial Hospital, Taiwan. There are 13 strains of monoclonal antibodies (mAbs) targeting on $\mathrm{S}$ protein, and 12 strains of $\mathrm{mAbs}$ targeting on $\mathrm{N}$ protein of the SARS-CoV-2. These antibodies especially those targeting on $\mathrm{S}$ protein can be a potential probe for the detection of S protein or the whole SARS-CoV-2 virus. The advantage of immune-based vial detection is its simplicity and speed (within 15 min). However, the antibodies prepared from SARS-CoV-2 protein antigen may cross-react with proteins from other similar viruses such as SARS-CoV and MERS, and thus a tedious screening procedure is needed before a specific testing kit can be developed.

\section{Viral Induced Proteins}

As aforementioned, the serology testing of COVID-19 is not targeting the virus itself but the antibodies such as IgM and IgG induced following viral infection. These immunoglobulins were serological testing markers for after the patient has early ( 3 to 6 days after exposure to the virus, $\operatorname{IgM}$ ) and late virus infection (after 8 days, IgG) response respectively [11]. The serological testing is crucial to identify active patients, 
asymptomatic carriers or convalescent persons and provide population surveillance. These data could be used to analyze and estimate the SARS-CoV-2 epidemiology and virology [12] and help to contain the outbreak of the pandemic. The highly reactive human could be the donor of convalescent serum for therapeutic [13]. The WHO interim guidance on COVID-19 testing suggested collecting blood, stool or autopsy materials such as lungs (deceased patients) as serology testing specimens [7]. As it has been reported that several factors could contribute to a high false-negative rate (FNR) when using NAAT, serology testing should also be taken into consideration as alternative testing or correlation testing [14]. There are companies such as Guangzhou Wondfo Biotech, China, Innovita Biological Technology, China; Pharma (Berlin), Germany and RayBiotech Inc. US have released various types of test kits that specifically testing on COVID-19 IgM and IgG.

\section{Summary and Perspective}

Specificity and sensitivity are two fundamental requirements for an effective diagnosis. It is also important that the testing method is user friendly. RNA detection is the most frequently used method for the identification of COVID-19 patients because this method is extremely sensitive due to the power of nucleic acid amplification and also highly specific by using complementary nucleic acid probe/primer for the identification of a particular RNA. The major drawback in such an emergency situation is probably the requirement of well-trained personnel and lengthy testing time (usually 3-4 hours) to run the test. However, NAAT has been announced that positive/negative COVID-19 can be determined in about 10 minutes by using the isothermal amplification method. This may due to the simplification of the NAAT procedure and the high amount of the SARS-CoV-2 at the beginning of the infection be detected.

Detection on viral proteins, such as $\mathrm{S}$ and $\mathrm{N}$ protein, give complementary information to that of RNA detection from the same sample. This method is more direct (without amplification), but is less sensitive. It needs more effort to obtain a suitable probe (usually antibody), but the testing method can be simple. Recent developments in biosensing technologies can significantly improve the protein sensing sensitivity such as the use of magnetic bead to concentrate the protein molecules or by applying nanowire field-effect transistor as a transducer. Many of the entrepreneurs may take this opportunity to fasten their product development. Serology testing targeting on viral-induced antibodies is given different information as those for viral RNA and proteins from SARS-CoV-2. Although the protein testing method is similar, the targets are not part of the virus and the testing specimens used can be quite different as compared to those for viral protein detection.

It is unfortunate that the world is currently suffering from the COVID-19 epidemic. However, we can expect that such an incident will re-appear. We wish that the world will be better prepared every time when we face the potential spread of an infectious disease. Biosensing technologies have progressed rapidly in the past decade. The pandemic outbreaks of COVID-19 continue to remind us that diagnostic tools are critical and needed to be prepared in advance.

\section{Acknowledgment}

This work was supported by the Ministry of Science and Technology, Taiwan ROC (Grant Numbers: 1082218-E-009-003).

\section{Conflict of interest}

The authors declare no financial or commercial conflict of interest.

\section{References}

1. Z. Wu, J. M. McGoogan, JAMA, 2020, 323, 1239.

2. World Health Organization, Coronavirus disease 2019 (COVID-19) Situation Report - 69 , 2020 .

3. Z. Y. Zu, M. D. Jiang, P. P. Xu, W. Chen, Q. Q. Ni, G. M. Lu, L. J. Zhang, Radiol ., 2020 , DOI: 10.1148/radiol.2020200490. 
4. D. J. Cleri, A. J. Ricketti, J. R. Vernaleo, Infect. Dis. Clin. North. Am ., 2010, $24,175$.

5. M. Mesel-Lemoine, J. Millet, P. O. Vidalain, H. Law, A. Vabret, V. Lorin, N. Escrios, M. L. Albert, B. Nal, F. Tangy, J. Virol .,2012, $86,7577$.

6. C. Wu, Y. Liu, Y. Yang, P. Zhang, W. Zhong, Y. Wang, Q. Wang, Y. Xu, M. Li, X. Li, M. Zheng, L. Chen, H. Li, Acta Pharm. Sin. B.,2020 , DOI: 10.1016/j.apsb.2020.02.008.

7. World Health Organization, Laboratory testing for coronavirus disease 2019 (COVID-19) in suspected human cases: interim guidance, 2020 .

8. V. M. Corman, O. Landt, M. Kaiser, R. Molenkamp, A. Meijer, D. K. W. Chu, T. Bleicker, S. Brünink, J. Schneider, M. L. Schmidt, D. G. J. C. Mulders, B. L. Haagmans, B. van der Veer, S. van den Brink, L. Wijsman, G. Goderski, J. L. Romette, J. Ellis, M. Zambon, M. Peiris, H. Goossens, C. Reusken, M. P. G. Koopmans, C. Drosten, Euro. Surveill.,2020 , 25 , DOI: 10.2807/1560-7917.ES.2020.25.3.2000045.

9. C. Sheridan, Nat. Biotechnol., 2020 , 38 , 382, DOI: 10.1038/d41587-020-00002-2.

10. C. Sheridan, Nat. Biotechnol., 2020 , DOI: 10.1038/d41587-020-00010-2.

11. Z. Li, Y. Yi, X. Luo, N. Xiong, Y. Liu, S. Li, R. Sun, Y. Wang, B. Hu, W. Chen, Y. Zhang, J. Wang, B. Huang, Y. Lin, J. Yang, W. Cai, X. Wang, J. Cheng, Z. Chen, K. Sun, W. Pan, Z. Zhan, L. Chen, F. Ye,J. Med. Virol., 2020 , DOI:10.1002/jmv.25727.

12. World Health Organization, Coronavirus disease 2019 (COVID-19) Situation Report - 59, 2020 .

13. K. Duan, B. Liu, C. Li, H. Zhang, T. Yu, J. Qu, M. Zhou, L. Chen, S. Meng, Y. Hu, C. Peng, M. Yuan, J. Huang, Z. Wang, J. Yu, X. Gao, D. Wang, X. Yu, L. Li, J. Zhang, X. Wu, B. Li, Y. Xu, W. Chen, Y. Peng, Y. Hu, L. Lin, X. Liu, S. Huang, Z. Zhou, L. Zhang, Y. Wang, Z. Zhang, K. Deng, Z. Xia, Q. Gong, W. Zhang, X. Zheng, Y. Liu, H. Yang, D. Zhou, D. Yu, J. Hou, Z. Shi, S. Chen, Z. Chen, X. Zhang, X.Yang, $P N A S$,2020 , DOI: 10.1073/pnas.2004168117.

14. Y. Wang, H. Kang, X. Liu, Z. Tong, J. Med. Virol.2020 , DOI: 10.1002/jmv.25721.

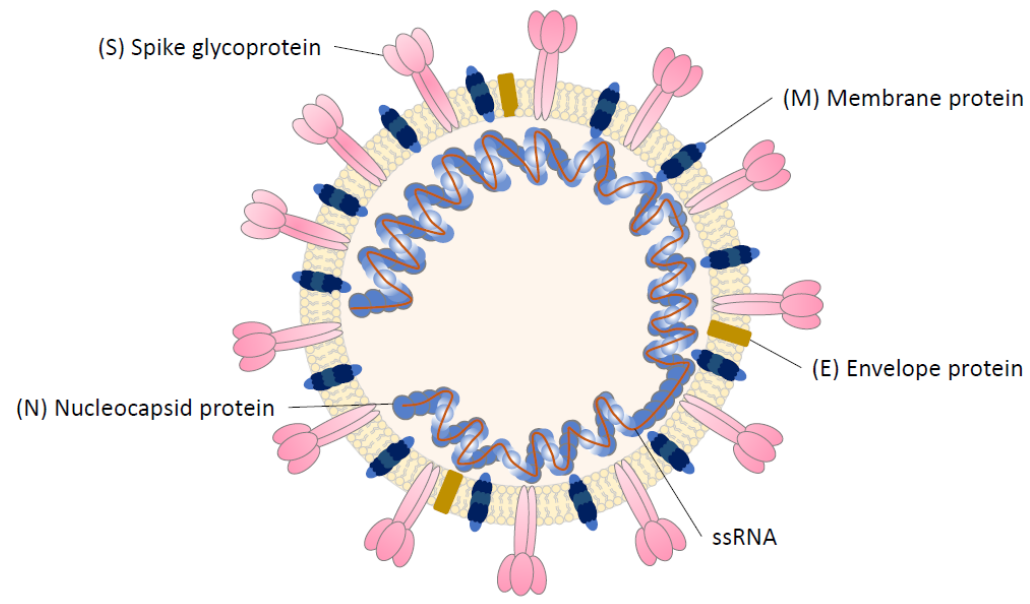

Figure 1. A typical structure of Betacoronaravirus virion.

\section{Hosted file}

Figure_1_Molecular Targets for the testing of COVID-19.pptx available at https://authorea.com/ users/312017/articles/442646-molecular-targets-for-the-testing-of-covid-19 